\title{
Sustained left ventricular diastolic dysfunction after exercise in patients with dilated cardiomyopathy
}

\author{
M Morikawa, H Sato, H Sato, Y Koretsune, Y Ohnishi, T Kurotobi, T Kuzuya, M Hori
}

\begin{abstract}
Objective-To investigate the recovery process of exercise induced diastolic dysfunction in heart failure, using Doppler echocardiographic techniques.

Design and patients-Transmitral flow velocity profiles and standard noninvasive haemodynamic indices were obtained serially over seven days after symptom limited bicycle exercise tests in 18 patients with dilated cardiomyopathy and eight normal subjects. In three patients with cardiomyopathy we also measured the pulmonary capillary wedge pressure for 24 hours after exercise.

Results-The intensity of exercise, as assessed by respiratory gas analysis, was lower in patients with dilated cardiomyopathy than in normal subjects. Despite the higher exercise level, all haemodynamic variables returned to baseline within one hour after exercise in normal subjects. In contrast, patients with dilated cardiomyopathy showed a sustained decrease in the peak early diastolic filling velocity and a sustained increase in the deceleration time of early filling for $\mathbf{2 4}$ hours or more after exercise. Because other haemodynamic variables recovered within one hour after exercise even in patients with dilated cardiomyopathy, the postexercise changes in ventricular filling were not explained by changes in loading conditions.
\end{abstract}

Conclusions-Exercise induced diastolic left ventricular dysfunction of the failing heart persists for 24 hours or more after exercise. The efficacy of exercise training on a daily basis in dilated cardiomyopathy requires further evaluation.

(Heart 1998;80:263-269)

Keywords: exercise; chronic heart failure; mitral flow velocity; diastolic stunning

Recent studies have shown unequivocally that exercise training for several months has beneficial effects on exercise tolerance, the autonomic nerve system, and the quality of life in patients with chronic heart failure. ${ }^{1-3}$ However, exercise activates neurohumoral factors, reduces renal blood flow, and perhaps precipitates cardiac arrhythmias. ${ }^{4-6}$ Moreover, exercise training may in itself have deleterious effects on the failing myocardium, because of the presence of several intrinsic abnormalities susceptible to exercise induced sympathetic stimula- tion and consequent tachycardia. ${ }^{7-12}$ Thus we cannot exclude the possibility that exercise training worsens long term prognosis in patients with chronic heart failure despite the short term benefits.

Diastolic ventricular function of the failing heart is reported to be impaired during exercise. ${ }^{13}$ We further showed that the exercise induced diastolic dysfunction is attenuated by $\beta$ adrenoceptor blockade. ${ }^{14}$ Because diastolic ventricular function is sensitive to transient myocardial metabolic insults, these findings suggest that exercise exerts a harmful metabolic effect on the failing myocardium through sympathetic stimulation. Moreover, the exercise induced diastolic dysfunction may persist despite cessation of exercise. Kono et al reported that the sympathetic activation during subarachnoid haemorrhage produces neurogenic myocardial stunning even in the normal heart. ${ }^{15}$ Thus even a physiological sympathetic activation during exercise may cause a sustained diastolic dysfunction in the failing heart because of its susceptibility to sympathetic stimulation. If this is the case, the harmful metabolic effects may accumulate during exercise training or even during daily physical activities, promoting progression of myocardial failure. Thus to determine a safe and logical exercise programme for patients with chronic heart failure it is important to clarify how long exercise induced diastolic dysfunction persists.

In this study, we investigated the recovery process of the exercise induced diastolic left ventricular dysfunction in patients with dilated cardiomyopathy, using reproducible Doppler echocardiographic techniques.

\section{Methods}

SUBJECTS

We studied 25 consecutive Japanese patients with chronic stable heart failure from dilated cardiomyopathy. All were in sinus rhythm. They had been admitted to hospital for evaluation. They were divided into two groups (table 1). In 18 patients, haemodynamic recovery was evaluated for seven days after exercise (group 1). In the remaining seven patients, the exercise test was not performed, although the same measurements were done to evaluate the day to day variability of haemodynamic indices (group 2). Because of cardiac enlargement the quality of transthoracic echocardiograms was good in all patients. Routine diagnostic cardiac catheterisation was performed within two weeks before the study in all patients. No significant coronary artery lesions or primary
Accepted for publication 12 May 1998 
Table 1 Patient characteristics

\begin{tabular}{llll}
\hline & \multicolumn{2}{l}{ Heart failure group } & \\
\cline { 2 - 3 } & Group 1 & Group 2 & Control group \\
\hline $\mathrm{n}$ & 18 & 7 & 8 \\
Age (years) & $53(13)$ & $49(12)$ & $46(14)$ \\
$\quad$ Range & 31 to 72 & 24 to 63 & 31 to 61 \\
Male/female & $13 / 2$ & $6 / 1$ & $7 / 1$ \\
NYHA (II/III) & $12 / 3$ & $6 / 1$ & \\
Cardiothoracic ratio (\%) & $56(7)$ & $56(5)$ & $48(4)$ \\
$\quad$ Range & 48 to 72 & 49 to 62 & 44 to 53 \\
LV ejection fraction (\%) & $31(8)$ & $26(9)$ & $66(10)$ \\
$\quad$ Range & 17 to 45 & 15 to 46 & 58 to 75 \\
LVEDV (ml) & $212(46)$ & $243(57)$ & $114(31)$ \\
$\quad$ Range & 181 to 299 & 191 to 375 & 84 to 140 \\
PCWP (mm Hg) & $11(8)$ & $14(11)$ & $6(3)$ \\
$\quad$ Range & 4 to 29 & 3 to 33 & 2 to 10 \\
Time interval between diagnostic & $6(4)$ & $7(4)$ & 5 (3) \\
$\quad$ catheterisation and the study (days) & $6(3)$ & & \\
\hline
\end{tabular}

LV, left ventricular; LVEDV, left ventricular end diastolic volume; NYHA, New York Heart Association functional class; PCWP, pulmonary capillary wedge pressure.

heart valve disease were detected in any patient. The left ventricular ejection fraction was assessed by contrast left ventriculography. All patients had been receiving diuretic agents, digitalis, and/or angiotensin converting enzyme inhibitors for more than three months before the study. During the study, these drugs were given once a day, with the morning meal at 08:00. No patient received $\beta$ blockers or inotropic agents, except for digitalis, during the study. The patient characteristics, including medication, were not significantly different between groups 1 and 2 (table 1 ).

To characterise the normal haemodynamic recovery after exercise, eight patients without organic cardiovascular disease were studied as a control group. Although they were somewhat younger than the patients with dilated cardiomyopathy, the difference was not significant. They had been admitted to hospital for evaluation of atypical chest pain. The presence of organic cardiovascular abnormalities was excluded by routine coronary arteriography, echocardiography, and exercise tests. No control subject was receiving any drug treatment during the study.

Written informed consent was obtained from all subjects before they entered the study. This study was approved by the hospital ethics committee.

STUDY PROTOCOL

On day 1, patients with dilated cardiomyopathy in group 1 and the control subjects were brought to the laboratory at 13:00 without eating lunch. After the subjects had rested for 10 minutes in the supine position, heart rate was measured with an electrocardiogram (ECG) monitor, and systemic blood pressure was measured with a sphygmomanometer. Baseline echocardiographic studies were then performed.

After baseline measurements were completed, subjects performed symptom limited upright bicycle exercise with one minute of unloaded pedalling, followed by ramp loading until they were nearly exhausted. The incremental load was set at $15 \mathrm{~W} / \mathrm{min}$ in patients with dilated cardiomyopathy and at $30 \mathrm{~W} / \mathrm{min}$ in the control subjects. Heart rate and blood pressure were measured every minute during exercise. Gas exchange data were collected throughout the exercise test using a breath by breath respiratory monitoring system (Minato Co, model RM-300, Tokyo, Japan). Oxygen uptake, carbon dioxide output, and the gas exchange ratio were determined as the mean of values obtained every 30 seconds.

To evaluate the recovery of left ventricular function after exercise, echocardiographic recordings and haemodynamic measurements were performed at 1,3 , and 5 hours and at 1,3 , and 7 days after exercise. The measurements at 1,3 , and 7 days after exercise were performed at 13:00 before lunch. Patients were allowed to rest for 10 minutes in the supine position before each evaluation. They were instructed to continue their ordinary sedentary routines in the hospital between measurements. Patients were allowed to eat the evening meal after all measurements were completed on day 1 . Water intake was unrestricted throughout the study.

Patients with dilated cardiomyopathy in group 2 followed their ordinary routines without performing the exercise test. Echocardiographic recordings and haemodynamic measurements were obtained as in group 1. The interval between the diagnostic catheterisation and the current study ranged from three days to two weeks.

\section{ECHOCARDIOGRAPHIC RECORDINGS}

$\mathrm{M}$ mode, cross sectional, and pulsed Doppler echocardiograms were recorded using an ultrasonic system (Toshiba Co, model SSH-160A, Tokyo, Japan) with a transducer array of 2.5 $\mathrm{mHz}$ and a pulse repetition rate of $4 \mathrm{kHz}$. M mode echocardiograms were recorded immediately below the anterior mitral valve leaflet during quiet respiration at a paper speed of 100 $\mathrm{mm} / \mathrm{s}$. An ECG and a phonocardiogram were recorded simultaneously.

The pulsed Doppler images were obtained in the apical four-chamber view. The transmitral flow velocity pattern was determined from the apex with the pulsed Doppler echocardiographic sample volume placed carefully between the tips of the mitral leaflets, where the maximum flow velocity in early diastole was obtained. All echocardiographic recordings were made by a well trained technician blinded to the purpose of the study. The recording conditions - including the timing of meals, the investigator, angle of the decubitus position, and the position and angle of the transducerwere also reproduced as exactly as possible throughout the study.

EVALUATION OF PULMONARY CAPILLARY WEDGE PRESSURE AND PLASMA VOLUME

The blood volume and left atrial pressure may decrease after exercise, affecting the transmitral flow velocity profile independently of myocardial factors. Hence we serially measured pulmonary capillary wedge pressure for 24 hours after exercise in three patients in group 1 from whom we were able to obtain informed consent for continuous right heart catheterisation. A $7 \mathrm{~F}$ catheter was advanced to the pulmonary artery one hour before the exercise test. Serial changes in blood volume were 
estimated using the following equation: blood volume $(\mathrm{A}) \div$ blood volume $(\mathrm{B})=$ haemoglobin (B) $\div$ haemoglobin (A). A and B refer to the value after exercise and that before exercise, respectively. ${ }^{16}$

DATA ANALYSIS

After all measurements were obtained, echocardiograms were coded and analysed by two investigators blinded to the patient and sequence of recordings. Left ventricular end diastolic and end systolic dimensions were obtained from $\mathrm{M}$ mode echocardiograms in the standard manner. ${ }^{17}$ Left ventricular fractional shortening was calculated using the following equation: $100 \times$ (end diastolic dimension - end systolic dimension)/end diastolic dimension.

Transmitral flow velocity profiles were analysed using a digitising pad interfaced with a computer system. We determined the peak early diastolic filling velocity (E), peak filling velocity at atrial contraction (A), and the deceleration time of the early diastolic flow velocity (fig 1). To avoid the influence of heart rate, the deceleration time was calculated as the time between the peak early diastolic filling velocity and the mitral deceleration slope extrapolated to the zero baseline. ${ }^{18}$ Cardiac cycles with a non-linear deceleration slope or in which early and late mitral flow velocities were fused were excluded from analysis. For each variable, five consecutive beats were measured, and the mean was used for analysis. The variability of flow velocity measurements was tested by repeated analysis of 17 pulsed Doppler echocardiograms. The standard deviation (SD) values for intraobserver measurements were $1.9 \mathrm{~cm} / \mathrm{s}$ for peak early diastolic filling velocity, $1.4 \mathrm{~cm} / \mathrm{s}$ for peak filling velocity

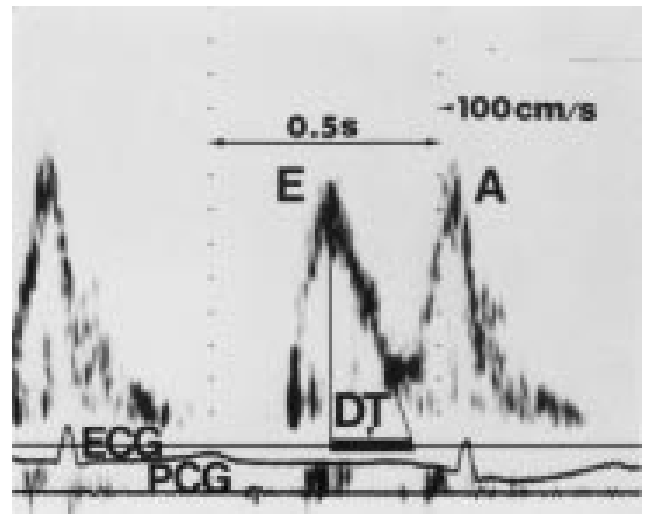

Figure 1 Pulsed Doppler mitral flow velocity tracings recorded in a patients with dilated cardiomyopathy. Three mitral flow velocity indices were measured: peak early diastolic filling velocity $(E)$, peak filling velocity at atrial contraction (A), and deceleration time (DT) of early diastolic filling velocity. ECG, electrocardiogram; PCG, phonocardiogram.

at atrial contraction, and $9.4 \mathrm{~ms}$ for deceleration time; the $\mathrm{SD}$ values for interobserver measurements of the same variables were 3.5 $\mathrm{cm} / \mathrm{s}, 1.8 \mathrm{~cm} / \mathrm{s}$, and $17.2 \mathrm{~ms}$, respectively.

\section{STATISTICAL ANALYSIS}

Data are expressed as the mean (SD). Intragroup comparisons were performed using a repeated measures analysis of variance (ANOVA) with multiple comparisons made by Bonferroni test, and intergroup comparisons were performed using ANOVA or the unpaired $t$ test as appropriate. ${ }^{19}$ A probability (p) value less than 0.05 was considered statistically significant.

\section{Results}

Baseline and postexercise haemodynamic data in each group are given in tables 2,3 , and 4 .

Table 2 Haemodynamic variables in the control group

\begin{tabular}{|c|c|c|c|c|c|c|c|c|}
\hline & \multirow[b]{2}{*}{ Baseline } & \multirow[b]{2}{*}{ Peak exercise } & \multicolumn{6}{|l|}{ Postexercise } \\
\hline & & & 1 hour & 3 hours & 5 hours & 1 day & 3 days & 7 days \\
\hline HR (beats/min) & 70 (13) & $168(5)$ & 73 (13) & 75 (11) & $74(11)$ & $70(11)$ & $74(11)$ & $71(11)$ \\
\hline SBP (mm Hg) & $117(7)$ & $210(8)$ & $117(6)$ & $120(9)$ & $121(7)$ & $119(7)$ & $121(9)$ & $121(8)$ \\
\hline $\operatorname{EDD}(\mathrm{mm})$ & $48(3)$ & & $48(3)$ & $47(3)$ & 47 (3) & 47 (3) & 47 (3) & 48 (3) \\
\hline $\operatorname{ESD}(\mathrm{mm})$ & $30(2)$ & & $29(2)$ & $29(1)$ & $30(2)$ & $29(2)$ & $29(2)$ & $29(2)$ \\
\hline FS (\%) & $38(4)$ & & $38(5)$ & $38(5)$ & $37(4)$ & $38(4)$ & $38(5)$ & $38(4)$ \\
\hline $\mathrm{E}(\mathrm{cm} / \mathrm{s})$ & $62(10)$ & & $60(9)$ & $61(11)$ & $60(9)$ & $62(10)$ & $63(7)$ & $63(10)$ \\
\hline $\mathrm{A}(\mathrm{cm} / \mathrm{s})$ & $51(7)$ & & $51(8)$ & $51(7)$ & $51(8)$ & $52(9)$ & $53(8)$ & $52(8)$ \\
\hline $\mathrm{E} / \mathrm{A}$ & $1.24(0.32)$ & & $1.23(0.33)$ & $1.23(0.35)$ & $1.22(0.32)$ & $1.23(0.32)$ & $1.21(0.27)$ & $1.24(0.32)$ \\
\hline $\mathrm{DT}(\mathrm{ms})$ & $161(18)$ & & $159(12)$ & $160(17)$ & $156(16)$ & $159(15)$ & $155(22)$ & $155(12)$ \\
\hline
\end{tabular}

Values are mean (SD). A, peak flow velocity at atrial contraction; DT, deceleration time of early diastolic filling; E, peak flow velocity of early diastolic filling; E/A, ratio of E to A; EDD, left ventricular end diastolic dimension; ESD, left ventricular end systolic dimension; FS, fractional shortening; HR, heart rate; SBP, systolic blood pressure.

Table 3 Haemodynamic variables in the heart failure group with exercise (group 1)

\begin{tabular}{|c|c|c|c|c|c|c|c|c|}
\hline & \multirow[b]{2}{*}{ Baseline } & \multirow[b]{2}{*}{ Peak exercise } & \multicolumn{6}{|l|}{ Postexercise } \\
\hline & & & 1 hour & 3 hours & 5 hours & 1 day & 3 days & 7 days \\
\hline HR (beats/min) & $74(11)$ & $142(8)$ & $72(15)$ & $71(9)$ & 73 (13) & $72(13)$ & $70(10)$ & $72(11)$ \\
\hline $\mathrm{SBP}(\mathrm{mm} \mathrm{Hg})$ & $117(16)$ & $163(17)$ & $117(22)$ & $116(17)$ & $118(16)$ & $119(19)$ & $118(18)$ & $118(18)$ \\
\hline $\operatorname{EDD}(\mathrm{mm})$ & $62(8)$ & & $61(8)$ & $62(9)$ & $62(8)$ & $62(8)$ & $62(8)$ & $62(8)$ \\
\hline $\operatorname{ESD}(\mathrm{mm})$ & $53(10)$ & & $52(11)$ & $53(10)$ & $53(11)$ & $53(10)$ & $53(10)$ & $53(10)$ \\
\hline FS $(\%)$ & $15(6)$ & & $15(6)$ & $15(6)$ & $15(6)$ & $15(6)$ & $15(5)$ & $16(6)$ \\
\hline $\mathrm{E}(\mathrm{cm} / \mathrm{s})$ & $48(6)$ & & $39(9) \dagger$ & $40(9) \dagger$ & $44(6)^{\star}$ & $43(7)^{\star}$ & $45(7)$ & $47(8)$ \\
\hline $\mathrm{A}(\mathrm{cm} / \mathrm{s})$ & $55(11)$ & & $59(9)$ & $59(10)$ & $60(8)$ & $55(9)$ & $56(9)$ & $57(10)$ \\
\hline $\mathrm{E} / \mathrm{A}$ & $0.91(0.25)$ & & $0.70(0.20) \dagger$ & $0.71(0.19) \dagger$ & $0.73(0.16) \dagger$ & $0.77(0.17) \dagger$ & $0.82(0.21)$ & $0.85(0.22)$ \\
\hline DT (ms) & $242(55)$ & & $276(58) \dagger$ & $284(67) \dagger$ & $282(71) \dagger$ & $278(61) \dagger$ & $251(60)$ & $247(47)$ \\
\hline
\end{tabular}

Values are means (SD). A, peak flow velocity at atrial contraction; DT, deceleration time of early diastolic filling; E, peak flow velocity of early diastolic filling; E/A, ratio of E to A; EDD, left ventricular end diastolic dimension; ESD, left ventricular end systolic dimension; FS, fractional shortening; HR, heart rate; SBP, systolic blood pressure.

${ }^{\star} \mathrm{p}<0.05 ; \mathrm{tp}<0.01 v$ baseline. 
Table 4 Haemodynamic variables in the heart failure group without exercise (group 2)

\begin{tabular}{|c|c|c|c|c|c|c|c|}
\hline & Baseline & 1 hour & 3 hours & 5 hours & 1 day & 3 days & 7 days \\
\hline HR (beats/min) & $71(14)$ & $70(13)$ & $71(15)$ & $72(13)$ & $71(13)$ & $72(15)$ & $74(12)$ \\
\hline $\mathrm{SBP}(\mathrm{mm} \mathrm{Hg})$ & $111(14)$ & $114(12)$ & $113(9)$ & $111(12)$ & $110(12)$ & $112(17)$ & $110(12)$ \\
\hline $\operatorname{EDD}(\mathrm{mm})$ & $63(9)$ & $63(10)$ & $63(9)$ & $62(8)$ & $62(8)$ & $63(9)$ & $63(9)$ \\
\hline $\operatorname{ESD}(\mathrm{mm})$ & $55(10)$ & $55(11)$ & $54(10)$ & $54(10)$ & $53(8)$ & $54(9)$ & $54(10)$ \\
\hline FS (\%) & $14(5)$ & $13(5)$ & $14(5)$ & $14(6)$ & $15(4)$ & $13(4)$ & $14(5)$ \\
\hline $\mathrm{E}(\mathrm{cm} / \mathrm{s})$ & $48(14)$ & $49(15)$ & $48(15)$ & $48(16)$ & $48(18)$ & $48(14)$ & 49 (15) \\
\hline $\mathrm{A}(\mathrm{cm} / \mathrm{s})$ & $56(18)$ & $58(20)$ & $57(18)$ & $57(19)$ & $56(19)$ & $58(20)$ & $57(19)$ \\
\hline $\mathrm{E} / \mathrm{A}$ & $0.94(0.46)$ & $0.94(0.46)$ & $0.94(0.45)$ & $0.95(0.50)$ & $0.96(0.50)$ & $0.94(0.50)$ & $0.96(0.50)$ \\
\hline DT (ms) & $245(19)$ & $246(18)$ & $243(19)$ & $245(14)$ & $243(19)$ & $245(13)$ & $244(17)$ \\
\hline
\end{tabular}

Values are means (SD). A, peak flow velocity at atrial contraction; DT, deceleration time of early diastolic filling; E, peak flow velocity of early diastolic filling; E/A, ratio of E to A; EDD, left ventricular end diastolic dimension; ESD, left ventricular end systolic dimension; FS, fractional shortening; HR, heart rate; SBP, systolic blood pressure.

BASELINE DATA

The baseline haemodynamic data in patients with dilated cardiomyopathy were almost identical between group 1 and group 2. Moreover, there were no significant differences in heart rate and blood pressure between the dilated cardiomyopathy groups and the control group at baseline. Left ventricular end diastolic and end systolic dimensions were enlarged and fractional shortening was reduced in the dilated cardiomyopathy groups. Peak early diastolic filling velocity (E) was less in the dilated cardiomyopathy groups than in the control group $(\mathrm{p}<0.05)$, whereas peak filling velocity at atrial contraction (A) did not differ significantly among the groups. Therefore the E/A ratio was reduced in the dilated cardiomyopathy groups compared with the control group $(p<0.05)$. The deceleration time of early diastolic filling was longer in the dilated cardiomyopathy groups than in the control group $(\mathrm{p}<0.05)$. The $\mathrm{E} / \mathrm{A}$ value exceeded 1.0 in four of 18 patients with dilated cardiomyopathy in group 1 (fig 2). Pulmonary capillary wedge pressure during the diagnostic cardiac catheterisation was markedly increased in two
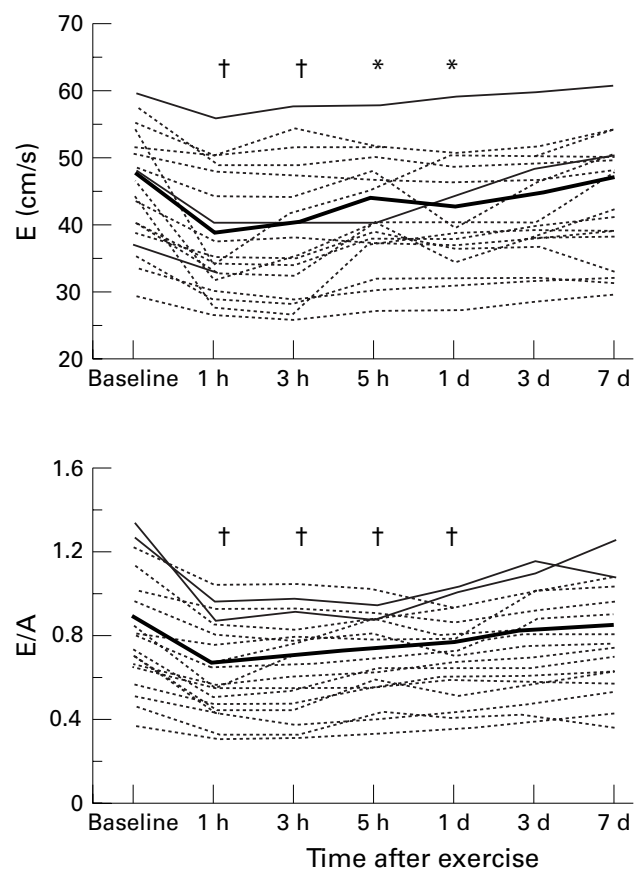

of the four patients $(29 \mathrm{~mm} \mathrm{Hg}$ and $25 \mathrm{~mm} \mathrm{Hg}$, respectively), suggesting that their $\mathrm{E} / \mathrm{A}$ values were pseudonormalised in association with raised left atrial pressures. In group 2, only one of seven patients had an E/A value above 1.0.

\section{EXERCISE DATA}

The duration of exercise was significantly shorter in group 1 than in the control group (table 5). At peak exercise, heart rate, systolic blood pressure, oxygen uptake, and the gas exchange ratio were also significantly less in group 1 than in the control group. Thus the intensity of exercise was less in group 1 than in the control group although the symptom limited near maximal exercise test was performed in both groups.

\section{POSTEXERCISE DATA}

There were no significant changes in any haemodynamic variable compared with baseline values throughout the postexercise period in the control group. These findings indicate that haemodynamic variables, including indices of diastolic function, normally returned to
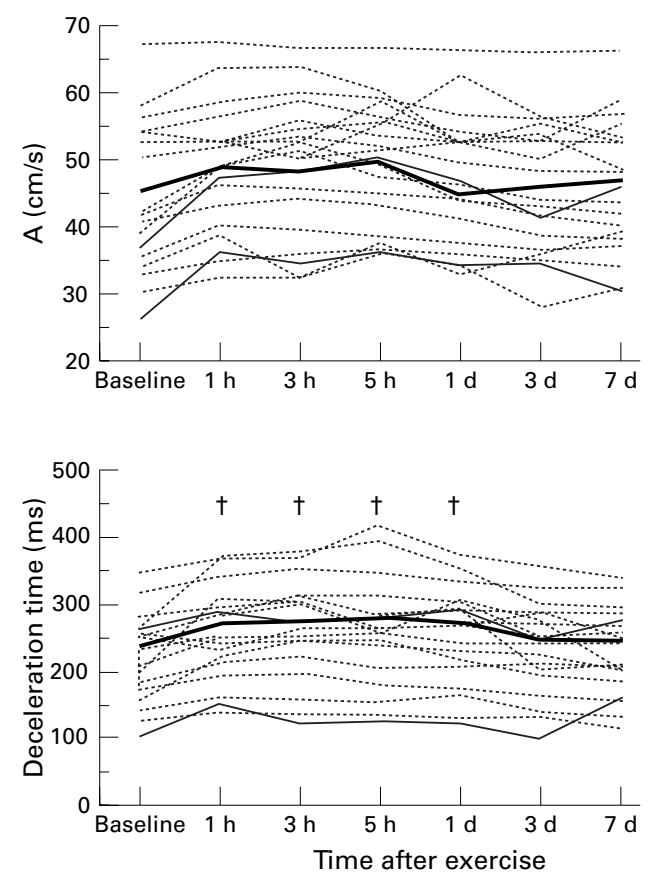

Figure 2 Postexercise changes in the peak early diastolic filling velocity (E), peak filling velocity at atrial contraction $(A)$, the ratio of $E$ to $A(E / A)$, and the deceleration time (DT) of early diastolic filling velocity in 18 patients with dilated cardiomyopathy. The thin solid and dashed lines indicate individual patients with and without pseudonormalisation of E/A, respectively. The thick line indicates the mean values. ${ }^{\star} p<0.05 ; \nmid p<0.01 v$ baseline. 
Table 5 Data at peak exercise

\begin{tabular}{lll}
\hline & $\begin{array}{l}\text { Heart failure } \\
\text { group 1 }\end{array}$ & $\begin{array}{l}\text { Control } \\
\text { group }\end{array}$ \\
\hline Exercise time (s) & $306(109)^{\star}$ & $394(57)$ \\
Peak HR (beats/min) & $142(8)^{\star}$ & $168(5)$ \\
Peak SBP (mm Hg) & $163(17)^{\star}$ & $210(8)$ \\
Peak $\dot{\mathrm{VO}}(\mathrm{ml} / \mathrm{min} / \mathrm{kg})$ & $16.1(2.6)^{\star}$ & $36.7(2.8)$ \\
Peak gas exchange ratio & $1.06(0.14)^{\star}$ & $1.22(0.07)$ \\
\hline
\end{tabular}

Values are means (SD). HR, heart rate; SBP, systolic blood pressure; $\dot{\mathrm{VO}}_{2}$, oxygen uptake.

${ }^{\star} \mathrm{p}<0.05 v$ control group.

baseline values within one hour after cessation of symptom limited maximum exercise.

In group 1, heart rate, systolic blood pressure, left ventricular dimensions, and fractional shortening also returned to baseline levels within one hour after exercise. However, E decreased for 24 hours after exercise, whereas A did not increase significantly during this period (fig 2). Therefore, the $\mathrm{E} / \mathrm{A}$ value decreased during this period. Moreover, the deceleration time of early diastolic filling was slightly but significantly increased for 24 hours after exercise. These variables returned to baseline values three days after exercise.

The postexercise changes in the mitral flow velocity pattern in group 1 were independent of the presence or absence of pseudonormalisa-
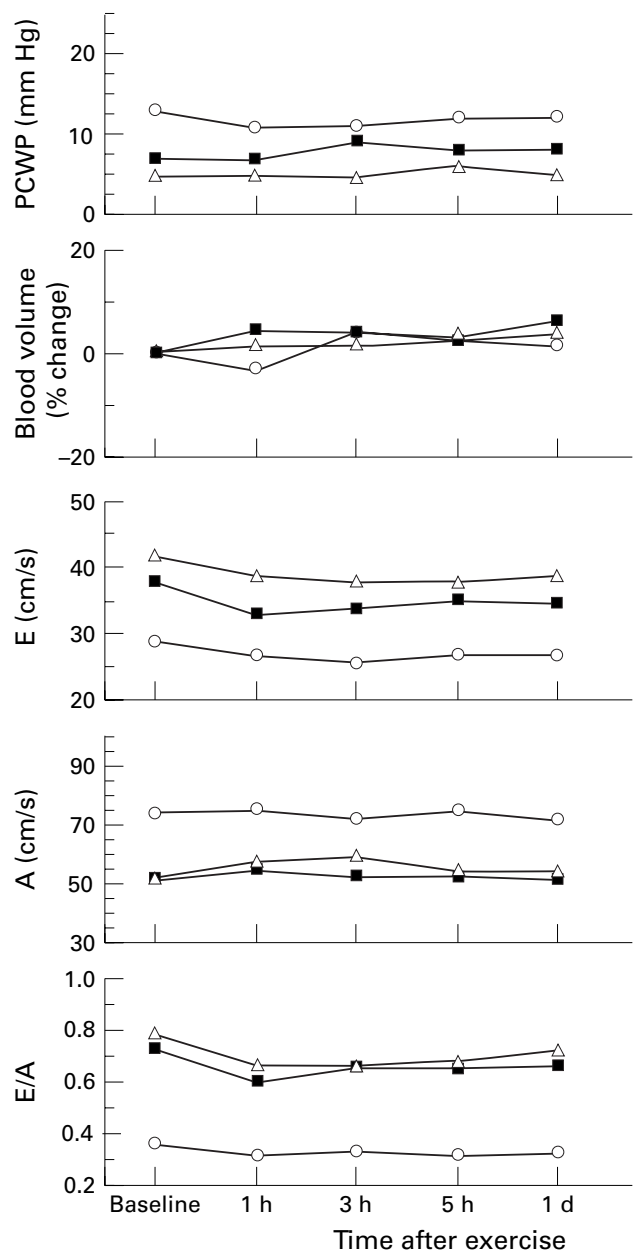

Figure 3 Postexercise changes in pulmonary capillary wedge pressure (PCWP), calculated blood volume, peak early diastolic filling velocity $(E)$, peak filling velocity at atrial contraction $(A)$, and the ratio of $E$ to $A(E / A)$ in three patients with dilated cardiomyopathy. tion of E/A at baseline (fig 2). There was also no significant relation between changes in the mitral flow velocity profile and other baseline haemodynamic variables.

The pulmonary capillary wedge pressure and blood volume were serially evaluated for 24 hours after exercise in three patients in group 1 . When compared with baseline values, neither variable showed a definite postexercise change in any patient despite sustained decreases in $\mathrm{E}$ and $\mathrm{E} / \mathrm{A}$ after exercise (fig 3 ).

REPRODUCIBILITY OF DOPPLER MEASUREMENTS In group 2, no significant changes were observed in any haemodynamic or Doppler echocardiographic variable throughout the study. E, A, and deceleration time values were highly reproducible over seven days.

\section{Discussion}

We limited the study population to heart failure patients with dilated cardiomyopathy to exclude the effect of transient myocardial ischaemia during exercise. Baseline mitral flow velocity profiles in our patients were consistent with those previously reported in patients with chronic heart failure with impaired left ventricular relaxation and raised left atrial pressure. ${ }^{18} 2021$ In the present study, patients with dilated cardiomyopathy showed a significant decrease in peak early diastolic filling velocity (E) and a non-significant increase in peak filling velocity at atrial contraction (A) for 24 hours after exercise. The deceleration time of early diastolic filling was increased during this period. These changes were not observed in the control group, even though control subjects achieved higher exercise levels than patients with dilated cardiomyopathy. Thus the postexercise alterations in the transmitral flow velocity pattern were characteristic responses of the failing heart.

\section{ALTERATIONS IN THE MITRAL FLOW VELOCITY} PROFILE

The major determinant of left ventricular early diastolic filling is the instantaneous transmitral pressure gradient. ${ }^{22}$ Therefore the postexercise decrease in $\mathrm{E}$ was attributed to a decrease in left atrial pressure, an increase in left ventricular early diastolic pressure, or both. Plasma volume is decreased during exercise as the result of a protein shift from the intravascular space to the extravascular space. ${ }^{23}$ However, the decreased plasma volume recovers completely within one hour, even after endurance exercise. ${ }^{23}$ In a previous study we also confirmed that the pulmonary capillary wedge pressure returned to baseline one hour after symptom limited bicycle exercise in patients with left ventricular dysfunction. ${ }^{24}$ Furthermore, the present study examined the serial changes in pulmonary capillary wedge pressure for 24 hours after exercise in three patients with dilated cardiomyopathy. As seen in fig 3, the pulmonary capillary wedge pressure did not show a definite change despite a sustained decrease in E. Thus it was unlikely that a hypovolaemic response and a subsequent reduction in left atrial pressure accounted for the postexercise decrease in $\mathrm{E}$. The decrease in 
E could therefore be attributed to a rise in left ventricular early diastolic pressure. Left ventricular early diastolic pressure is determined by several factors, including the loading conditions, diastolic elastic recoil, and the rate of relaxation. ${ }^{25-28}$ In the present study, blood pressure, left ventricular dimensions, and systolic function assessed by the fractional shortening were unchanged throughout the postexercise period. Therefore the decrease in $\mathrm{E}$ observed during this period is explained neither by changes in the loading condition nor by a decrease in elastic recoil. It was most plausible that the decrease in $\mathrm{E}$ was a reflection of postexercise abnormalities of left ventricular relaxation. The insignificant increase in A observed after exercise in patients with dilated cardiomyopathy may have been a compensatory mechanism in response to the decrease in $\mathrm{E}$.

There are two possible mechanisms of the postexercise increase in the deceleration time of early diastolic filling observed in patients with dilated cardiomyopathy: an increase in passive chamber distensibility and a decrease in the relaxation rate. ${ }^{18}$ However, we have previously shown that left ventricular chamber distensibility was unchanged or even decreased during exercise in patients with dilated cardiomyopathy. ${ }^{14}$ Thus the increase in deceleration time may also have been related to exercise induced abnormalities of left ventricular relaxation.

MECHANISM OF POSTEXERCISE DIASTOLIC DYSFUNCTION

Diastolic left ventricular function deteriorates during exercise in patients with chronic heart failure. ${ }^{14}$ The present study strongly suggests that this abnormality persists for 24 hours or more after cessation of exercise. The underlying cause of this deterioration remained unclear in this study. However, several intrinsic abnormalities susceptible to exercise induced sympathetic stimulation and consequent tachycardia are known in the failing heart. The failing heart has reduced coronary vasodilator reserve. $^{7}$ This abnormality may cause an energy depleted state during exercise. It has been suggested that a decrease in the left ventricular relaxation rate is a reflection of abnormal calcium handling, such as reduced calcium sequestration by the sarcoplasmic reticulum. ${ }^{91112}$ Moreover, heart rate dependent potentiation of relaxation is markedly impaired in failing hearts. ${ }^{78}$ In the presence of these abnormalities, sympathetic mediated increases in the cytosolic calcium transit and shortening of diastole may outweigh the sympathetic mediated or frequency dependent acceleration of the deactivation process, resulting in accumulation of cytosolic calcium during diastole. This abnormality may produce long lasting ventricular dysfunction, as suggested by previous studies on reversible postischaemic dysfunction known as "stunned myocardium". ${ }^{29-34}$

Exercise induced oxidative stress may be another possible mechanism of sustained diastolic dysfunction. Kumar et al reported that endurance exercise in adult rats increased the generation of free radicals and lipid peroxida- tion in the myocardium..$^{35}$ However, it is unclear whether brief exercise for several minutes employed in this study causes myocardial dysfunction through oxidative stress.

Further studies are needed to clarify the mechanism of sustained left ventricular diastolic dysfunction after exercise.

\section{STUDY LIMITATIONS}

Although the exercise test was performed after diagnostic catheterisation after an interval of more than three days, non-specific effects of catheterisation and contrast medium might modify postexercise diastolic function. However, the interval between the diagnostic catheterisation and exercise test did not differ significantly among the groups (table 1 ). Thus the postexercise diastolic dysfunction in group 1 could not be attributed to the effect of catheterisation.

We performed echocardiography serially over a seven day period after exercise. Therefore it is possible that postexercise changes in Doppler indices in patients with dilated cardiomyopathy were a result of measurement errors or day to day variability. However, the mitral flow velocity profile was unchanged throughout the study in the normal subjects and in the dilated cardiomyopathy patients in group 2. Thus day to day variability of Doppler measurements were minimal. Bias related to echocardiographic analysis was also excluded, as the investigators were blinded to the sequence of recordings. It is thus unlikely that the changes in Doppler indices were a result of measurement errors.

Our patients with dilated cardiomyopathy had been receiving diuretic agents, digitalis, and angiotensin converting enzyme inhibitors. Although the measurements were performed five hours after the administration of drugs, it is possible that postexercise changes in the mitral flow velocity pattern were due, partly, to the acute effects of these drugs. However, the flow velocity changes observed 24 hours after exercise were independent of drug effects because the interval between drug administration and the Doppler measurements was set identically at baseline and after exercise. However, we cannot completely exclude chronic effects of the drugs, especially digitalis. This drug may potentiate the deleterious effects of exercise on the failing myocardium by influencing intracellular calcium handling. However, as all our patients in group 1 had been treated with digitalis, we could not perform subgroup analysis. Further studies are needed to clarify the interactive effect of exercise and digitalis on failing hearts.

Normal subjects served as controls in this study. Therefore it is unclear whether the postexercise diastolic dysfunction is characteristic of the failing heart or is a non-specific effect of cardiac enlargement. However, we previously showed that diastolic function does not deteriorate during exercise in patients with aortic regurgitation and dilated but non-failing hearts. ${ }^{14}$ Thus it is unlikely that postexercise diastolic dysfunction in patients with dilated cardiomyopathy was caused by cardiac enlargement. 
However, the cause of the heart failure in the present study was dilated cardiomyopathy. Further studies are needed to clarify whether sustained postexercise diastolic dysfunction is observed in other forms of chronic heart failure.

CLINICAL IMPLICATIONS

Our study showed that in patients with dilated cardiomyopathy, left ventricular diastolic dysfunction caused by intense exercise is sustained over 24 hours or more despite cessation of exercise. This finding may explain how heart failure is aggravated by repetitive exercise but improved by rest. ${ }^{36}$ If a patient with chronic heart failure performs exercise at short intervals - for example, on a daily basis, diastolic function may deteriorate cumulatively and further cardiac dysfunction may result.

Our results have important implications for exercise programmes in patients with heart failure. They do not directly controvert the beneficial effects of physical exercise in patients with chronic heart failure because most rehabilitation programmes use a less intense exercise protocol than that used in our study. However, sympathetic activation that may account for exercise induced diastolic dysfunction is inevitable even during submaximal exercise in patients with chronic heart failure. ${ }^{37}$ Although further studies are needed to assess the optimal amount of exercise in patients with chronic heart failure, our results imply that an adequate interval between exercise episodes is essential to reduce the risk of progression of heart failure during long term exercise training.

1 Koch M, Douard H, Broustet JP. The benefit of graded physical exercise in chronic heart failure. Chest 1992;104:231-5S.

2 Coats AJS, Adamopoulos S, Radaelli A, et al. Controlled trial of physical training in chronic heart failure: exercise performance, hemodynamics, ventilation, and autonomic function. Circulation 1992;85:2119-31

3 Sullivan MJ, Higginbotham MB, Cobb FR. Exercise training in patients with severe left ventricular dysfunction: hemodynamic and metabolic effects. Circulation 1988;78 506-15.

4 Drexler H. Reduced exercise tolerance in chronic heart failure and its relationship to neurohumoral factors. Eur Heart f 1991;12 (suppl C):21-8.

5 Gleim GW, Zabetakis PM, Depasquale EE, et al. Plasma osmolality, volume, and renin activity at the "anaerobic osmolality, volume, and renin activity at

6 Muller AF, Batin P, Evans S, et al. Regional blood flow in chronic heart failure: the reason for the lack of correlation chronic heart failure: the reason for the lack of correlation between patients' exercise
Heart F 1992;67:478-81.

7 Knecht M, Burkhoff D, Yi GH, et al. Coronary endothelial dysfunction precedes heart failure and reduction of coronary reserve in awake dogs. $\mathcal{F}$ Mol Cell Cardiol 1997;29:21727.

8 Mulieri LA, Hasenfuss G, Leavitt B, et al. Altered myocardial force-frequency relation in human hear failure. Circulation 1992;85:1743-50.

9 Gwathmey JK, Copelas L, MacKinnon R, et al. Abnormal intracellular calcium handling in myocardium from pa-
tients with end-stage heart failure. Circ Res 1987;61:70-6.

10 Gilbert JC, Glantz SA. Determinants of left ventricular filling and of the diastolic pressure-volume relation. Circ Res 1989;64:827-52.

11 Whitmer JT, Kumar P, Salaro RJ. Calcium transport properties of cardiac sarcoplasmic reticulum from cardio-
myopathic Syrian hamsters (BIO 53.58 and 14.6): myopathic Syrian hamsters (BIO 53.58 and 14.6):
evidence for a quantitative defect in dilated myopathic hearts not evident in hypertrophic hearts. Circ Res 1988;62: $81-5$.
12 Beuckemann DJ, Nabauer M, Erdmann E. Intracellular calcium handling in isolated ventricular myocytes from patients with terminal heart failure. Circulation 1992;85: 1046-55.

13 Cheng CP, Noda T, Nozawa T, et al. Effect of heart failure on the mechanism of exercise-induced augmentation of mitral valve flow. Circ Res 1993;72:795-806.

14 Sato H, Hori M, Ozaki H, et al. Exercise-induced upward shift of diastolic left ventricular pressure-volume relation in patients with dilated cardiomyopathy: effects of
$\beta$-adrenoceptor blockade. Circulation 1993;88:2215-23.

15 Kono T, Morita H, Kuroiwa T, et al. Left ventricular wall motion abnormalities in patients with subarachnoid hemorrhage: neurogenic stunned myocardium. $\mathcal{F} \mathrm{Am}$ Coll Cardiol 1994;24:636-40.

16 Dill DB, Costill DL. Calculation of percentage changes in volume of blood, plasma, and red cells in dehydration. $\mathcal{F}$ Appl Physiol 1974;37:247-8.

17 Sahn DJ, DeMaria A, Kisslo J, et al. Recommendations regarding quantitation in M-mode echocardiography: results of a survey of echocardiographic measurements. Circulation 1978;58:1072-83.

18 Appleton CP, Hatle LK, Popp RL. Relation of transmitral flow velocity patterns to left ventricular diastolic function: new insights from a combined hemodynamic and Doppler echocardiographic study. $\mathcal{F}$ Am Coll Cardiol 1988;12:42640.

19 Snedecor GW, Cochran WG. Statistical methods, 7th ed. Ames, Iowa; Iowa State University Press, 1980

20 Takenaka K, Dabestani A, Gardin JM, et al. Pulsed Doppler echocardiographic study of left ventricular filling in dilated cardiomyopathy. Am f Cardiol 1986;58:143-7.

21 Vanoverschelde JLJ, Raphael DA, Robert ARR, et al. Left ventricular filling in dilated cardiomyopathy: relation to functional class and hemodynamics. F $\mathrm{Am}$ Coll Cardiol 1990;15:1288-95.

22 Ishida Y, Meisner JS, Tsujioka K, et al. Left ventricular filling dynamics: influence of left ventricular relaxation and ing dynamics: influence of left ventricular rela

23 Harrison MH, Edwards RJ, Leitch DR. Effect of exercise and thermal stress on plasma volume. $\mathcal{F}$ Appl Physiol 1975; 39:925-31.

24 Sato $\mathrm{H}$, Inoue $\mathrm{M}$, Matsuyama $\mathrm{T}$, et al. Hemodynamic effects of the $\beta_{1}$-adrenoceptor partial agonist xamoterol in relation to plasma norepinephrine levels during exercise in patients with left ventricular dysfunction. Circulation 1987;75:21320.

25 Cheng CP, Freeman GL, Santamore WP, et al. Effect of loading conditions, contractile state, and heart rate on early diastolic left ventricular filling in conscious dogs. Circ Res 1990;66:814-23.

26 Yellin EL, Nikolic S, Frater RWM. Left ventricular filling dynamics and diastolic function. Prog Cardiovasc Dis 1990; 32:247-71

27 Little WC, Downes TR. Clinical evaluation of left ventricular diastolic performance. Prog Cardiovasc Dis 1990;32: 273-90.

28 Yamamoto K, Masuyama T, Tanouchi J, et al. Importance of left ventricular minimal pressure as a determinant of transmitral flow velocity pattern in the presence of left ventricu-
lar systolic dysfunction. $\mathcal{F}$ Am Coll Cardiol 1993;21:662-72.

29 Braunwald E, Kloner RA. The stunned myocardium: prolonged, post ischemic ventricular dysfunction. Circulaprolonged, post ischemi
tion $1982 ; 66: 1146-9$.

30 Kusuoka H, Porterfield JK, Weisman HF, et al. Pathophysiology and pathogenesis of stunned myocardium. Depressed $\mathrm{Ca}^{2+}$ activation of contraction as a consequence of reperfusion-induced cellular calcium overload in the ferret heart. F Clin Invest 1987;79:950-61

31 Marban E, Koretsune Y, Corretti M, et al. Calcium and its role in myocardial cell injury during ischemia and reperfusion. Circulation 1989;80(suppl IV):IV-17-22.

32 Kitakaze M, Weisman HF, Marban E. Contractile dysfunction and ATP depletion after transient calcium overload in perfused ferret hearts. Circulation 1988;77:685-95.

33 Koretsune Y, Marban E. Cell calcium in the pathophysiology of ventricular fibrillation and in the pathogenesis of ogy of ventricular fibrillation and in the pathogenesis of
postarrhythmic contractile dysfunction. Circulation 1989; po:369-79.

34 Kitakaze M, Weisfeldt ML, Marban E. Acidosis during early reperfusion prevents myocardial stunning in perfused ferret hearts. $\mathcal{F}$ Clin Invest 1988;82:920-7.

35 Kumar CT, Reddy VK, Prasad M, et al. Dietary supplementation of vitamin $\mathrm{E}$ protects heart tissue from exerciseinduced oxidant stress. Mol Cell Biochem 1992;111:109-15.

36 Burch GE, Walsh JJ, Black WC. Value of prolonged bed rest in management of cardiomegaly. $\mathcal{F} A M A$ 1963;183:81-7.

37 Ozaki H, Sato H, Hori $\mathrm{M}$, et al. Predominant $\beta$-adrenoceptor blocking effect of xamoterol averaged over the day in patients with mild to moderate heart failure: insight into the mechanism of its long-term clinical efficacy. Eur f Clin Pharmacol 1992;43:455-61. 